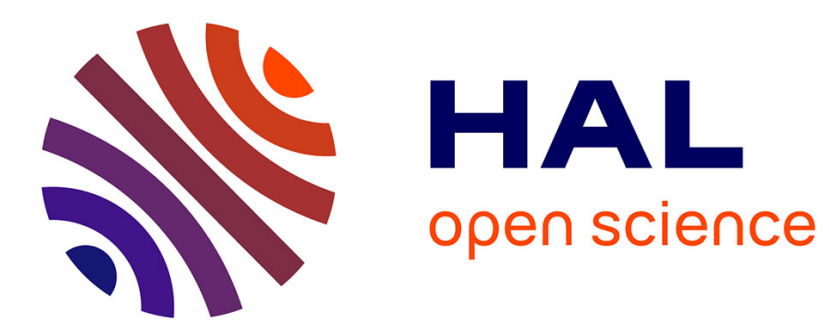

\title{
La formation des cadres de la bureaucratie en Autriche-Hongrie
}

\author{
Julia Bavouzet
}

\section{To cite this version:}

Julia Bavouzet. La formation des cadres de la bureaucratie en Autriche-Hongrie. Savoirs et métiers de l'Etat au XIXe siècle, Peter Lang AG, 2014, 10.3726/978-3-0352-0270-0/21 . hal-02075784

\section{HAL Id: hal-02075784 \\ https://hal.science/hal-02075784}

Submitted on 21 Mar 2019

HAL is a multi-disciplinary open access archive for the deposit and dissemination of scientific research documents, whether they are published or not. The documents may come from teaching and research institutions in France or abroad, or from public or private research centers.
L'archive ouverte pluridisciplinaire HAL, est destinée au dépôt et à la diffusion de documents scientifiques de niveau recherche, publiés ou non, émanant des établissements d'enseignement et de recherche français ou étrangers, des laboratoires publics ou privés. 


\title{
La formation des cadres de la bureaucratie en Autriche-Hongrie
}

\author{
Julia Bavouzet \\ Centre de Recherches Pluridisciplinaires Multilingues (EA 4418), Université Paris Ouest Nanterre la Défense, \\ France
}

\section{Résumé}

L'ouvrage aborde dans une optique comparatiste (France, Allemagne) les modalités de formation, de recrutement et d'évolution de carrière des serviteurs de l'Etat au XIXe siècle, dans un contexte marqué par la construction d'un Etat moderne soucieux d'une gestion efficace. A la différence de la Prusse, cas emblématique et précoce d'Etat autoritaire-bureaucratique, l'organisation d'une fonction publique régulière en France a en large partie résulté de nécessités pratiques et non d'une doctrine positive. Toutefois, les qualifications nécessaires pour que les administrateurs soient reconnus compétents et légitimes ont fait l'objet de vifs débats, traversés par des phénomènes de circulations d'idées et de modèles.

Les pratiques nées des nécessités du service de l'Etat ont permis des assouplissements et adaptations qui tempèrent les contrastes offerts par les représentations idéal-typiques du bureaucrate allemand et de son homologue français, contrastes qui renvoient à des traditions différentes en matière de relations entre pouvoir politique et administration. Au-delà de la diversité des situations, c'est la question du lien entre le pouvoir administratif et la poursuite de l'intérèt général qui demeure tout à la fois sous-jacente et active.

Cette contribution se propose d'étudier la formation des cadres de la bureaucratie ministérielle en Autriche-Hongrie dans la seconde moitié du long XIXe siècle. Il sera en effet question de l'évolution de la formation universitaire dans une logique de modernisation et de professionnalisation de l'enseignement des sciences administratives. Cette évolution se fond dans le mouvement plus général d'émergence d'une bureaucratie d'Etat qui se formalise tout au long de la période.

Il faut avant tout rappeler que la seconde moitié du XIXe siècle fut pour la Hongrie une période de grands bouleversements politiques. La forme même de l'Etat fut plusieurs fois modifiée, ce qui entraîna de profonds changements dans le personnel administratif. Dominée par l'Autriche des Habsbourg depuis la défaite des Ottomans au XVIe siècle, ce qui la place dans l'aire culturelle germanique, la Hongrie perd en effet toute indépendance et se voit directement administrée depuis Vienne après l'échec de la révolution et de la guerre d'indépendance de 1848. C'est la période noire du néo-absolutisme et de l'administration par les «hussards de Bach», dont les conséquences sont décisives tant pour le monde universitaire que pour la formation de l'appareil d'Etat. Au contraire, après le Diplôme d'octobre (1861), les rapports se normalisent entre Vienne et Budapest et la période est marquée par une détente, préambule au modus vivendi inauguré par le Compromis de 1867. Cette nouvelle situation est alors inédite : la Hongrie jouit d'une autonomie quasi totale dans la Double Monarchie qui se réalise par la nomination d'un gouvernement indépendant, responsable devant le Parlement de Budapest. C'est depuis ce moment que l'on peut parler au sens strict d'une «bureaucratie ministérielle» et 
d'une «bureaucratie d'Etat» hongroise. On verra alors comment se traduit cette indépendance dans la formation des hauts fonctionnaires de l'administration centrale ${ }^{1}$.

Cette époque fut celle de la rationalisation de l'appareil d'Etat, menée par les pouvoirs successifs. La formalisation et la codification du statut de fonctionnaire n'ont pourtant pas abouti à la veille de la première guerre mondiale. Le politique n'est pas encore différencié du champ administratif au sens propre, le vocabulaire aussi est incertain : des variations dans la terminologie se retrouvent dans les études, qui parfois ne recoupent pas la même réalité (par exemple pour l'administration : «közszolgálat», «közigazgatás», «beligazgatás» et «adminisztráció», ou, pour le fonctionnaire : «hivatalnok» qui tend à être remplacé par «tisztviselö», «állami tisztviselö» et «köztisztviselö» ${ }^{2}$ ). La fixation du vocabulaire relève de la logique de codification et de professionnalisation qui anime, plus généralement, la rationalisation de l'appareil d'Etat.

En ce qui concerne la formation de l'élite bureaucratique, cette rationalisation passe par le biais de réformes successives, concernant tant l'élargissement et la modernisation du réseau de l'offre de formation que la spécialisation et la technicisation de l'enseignement de sciences politiques, et finalement la codification de la qualification. Celle-ci débuta sous Marie-Thérèse lorsqu'elle assigna à la Faculté de droit le rôle de former l'élite des fonctionnaires et y introduisit l'enseignement des sciences camérales : une formation universitaire était donc requise des hauts fonctionnaires. C'est le début d'une formalisation de plus en plus poussée de la formation par le droit de l'élite administrative, qui aboutit en 1883 à la loi dite de «qualification», imposant le «privilège des juristes».

\section{Les différents parcours de formation}

Une première approche de la formation des hauts fonctionnaires de l'administration centrale consiste à dresser un tableau de l'offre de formation universitaire et académique, afin de mettre à jour les hauts lieux de formation. Si l'état actuel des recherches ne permet pas d'avoir d'informations précises ${ }^{3}$, on peut tout de même estimer que la grande majorité (plus de 90\%) du personnel des ministères et des cabinets, des hauts fonctionnaires et des députés étaient passés par la filière du droit. En effet, il n'existait pas en Hongrie d'établissements réservés à la formation des hauts fonctionnaires, à l'instar du Theresianum et de la Konsularakademie de Vienne. Ces établissements d'élite, sous direction de l'Etat, pratiquant l'exclusivisme nobiliaire, furent fondés au milieu du XVIIIe siècle dans le but d'offrir une formation pratique à l'élite bureaucratique, hors de l'Université jugée trop bourgeoise et destinée idéalement à former des fonctionnaires de moindre rang ${ }^{4}$. Cette dualité du système de formation entre l'Uni-

1. Cette étude se restreint en effet aux hauts fonctionnaires de l'administration centrale et laisse volontairement de côté l'administration locale des comitats dont les modalités sont radicalement différentes. Cette profonde dualité de l'administration centrale/locale est une particularité de la Hongrie. L'administration locale est très étudiée comme étant une particularité nationale, puisqu'elle revendique d'être le bastion de l'identité hongroise. En revanche, l'administration centrale l'est moins. Pour ce qui est de l'administration locale des comitats, voir l'étude de référence de G. BARANY, Ungarns Verwaltung : 1848-1918, in Die Habsburgermonarchie, Verlag der österreichischen Akademie der Wissenschaften, 1973.

2. J. CSIKVARY, A Tisztviselői Mozgalmak Története. 1867-1908 Budapest, 1909.

3. Le dépouillement systématique des CV (minôsítési lapok) des fonctionnaires en vue d'établir leurs études et leurs diplômes n'a pas encore été effectué. Ce manque est à replacer dans celui, plus général, d'études sur les hauts fonctionnaires.

4. Seuls 5\% des plus hauts fonctionnaires (Spitzenbeamten) sont issus de l'université. Voir G. STIMMER, Eliten in österreich 1848-1970, Böhlau, Wien, 1997. 
versité d'un côté, et les établissements d'Etat élitaires de l'autre, n'existait pas en Hongrie, où l'université, et plus précisément la faculté de droit, remplissait seule le rôle de formation des hauts fonctionnaires. Cette étude se concentre donc sur l'offre de formation par le droit, à défaut de pouvoir suivre les parcours réels.

Il faut avant tout mentionner la Faculté de droit de l'université de Budapest, qui occupait une place centrale dans le marché des études supérieures. Il s'agissait de la seule université du pays, la plus importante même après 1872 lorsque s'ouvrit une seconde université. La Faculté de droit y était de loin la plus développée, jusqu'à l'hypertrophie : à Budapest en 1870, on comptait 1274 étudiants en droit, c'est-à-dire $65 \%$ des étudiants de la capitale ${ }^{5}$. Ce chiffre est à resituer dans le cadre d'une surproduction générale de juristes en Hongrie tout au long du XIXe siècle : jusqu'au tournant du siècle, plus de la moitié des étudiants du pays étaient inscrits en droit ${ }^{6}$, au point que le terme de «jurátusz» devint synonyme d'étudiant. Si la Faculté de Budapest resta tout au long de la période le premier centre de formation des juristes par le nombre, elle le fut aussi par la qualité : contrairement aux académies de droit, elle pouvait délivrer le plus haut diplôme de l'éducation supérieure, à savoir le doctorat, et son enseignement restait de meilleure qualité. On peut donc supposer (à défaut d'estimations chiffrées) que le passage par la Faculté de droit de Budapest constituait la «voie royale» menant aux bureaux de l'administration centrale.

La Faculté de droit de l'université de Kolozsvár, fondée en 1872 pour répondre à l'engorgement de l'université de Budapest ${ }^{7}$, vint concurrencer la première : au tournant du siècle, elle accueille déjà un quart des étudiants en droit du pays, à savoir un millier d'étudiants contre plus de 3800 à Budapest ${ }^{8}$. Mais elle resta toujours à une place secondaire, notamment à cause du faible niveau de son enseignement. On peut en avoir une preuve dans l'inflation des diplômes délivrés : trois fois plus qu'à Budapest (837 diplômes en 1905 à Kolozsvár contre 281 dans la capitale ${ }^{9}$ ), quand la nouvelle université accueillait alors trois fois moins d'étudiants. Cette «fabrique de doctorats» permettait aux étudiants de mettre en place une stratégie de meilleur rendement de leurs études, particulièrement visible dans les facultés de droit : la première année se faisait à Budapest, afin de profiter de la sociabilité offerte par la capitale, puis les étudiants migraient à Kolozsvár où ils obtenaient leur diplôme au prix d'un moindre investissement intellectuel. Comme nous le verrons, cette pratique entravait l'exigence d'excellence intellectuelle dans les facultés de droit.

La fondation de cette nouvelle Faculté de droit à Kolozsvár permit de répondre à une plus grande demande en formation supérieure, mais l'université ne pouvait à elle seule former la masse des fonctionnaires dans le contexte d'augmentation du personnel administratif et de l'appareil d'Etat au XIXe siècle. En effet, il faut rappeler qu'avec la victoire de l'idée de centralisation, c'est-à-dire de l'élargissement des prérogatives de l'administration centrale sur l'ad-

5. Chiffres tirés de l'Annuaire statistique de la Hongrie, 1872, cités par V. KARADY, Une «nation de juristes», Actes de la recherche en sciences sociales, 1991.

6. A. LADANYI, A magyarországi felsőoktatás a dualizmus kora második felében, Felső ktatási pedagógiai Kutatóközpont, Budapest, 1969.

7. Il y avait en 1870 à Budapest 1336 étudiants en droit, chiffre qui diminue à l'ouverture de l'université de Kolozsvár (1278 étudiants en 1874), mais continue d'augmenter par la suite (1455 étudiants en 1880, 1786 en 1890, et 3814 en 1900). Chiffres tirés de F. ECKHART, A Jog-Es Allamtudományi Kar Története, 1667- 1935 Királyi Magyar Egyetemi Nyomda, 1936.

8. Ibidem.

9. A. LADANYI, A magyarországi felsőoktatás a dualizmus kora második felében, op. cit. 
ministration locale, et la création de nouveaux secteurs comme le chemin de fer, le télégraphe et le téléphone, l'électricité, etc., les besoins en personnel d'Etat qualifié (notamment aux postes centraux) croissaient de manière exponentielle. Quelques chiffres : dans les années 1860, il y avait environ 16000 employés de l'Etat (sans compter le personnel administratif des comitats). Au tournant du siècle, ils étaient 98 000, et 229000 en $1918^{10}$. Cet accroissement entraîna l'élargissement et la restructuration de l'offre de formation, par des interventions directes de l'Etat, par exemple la fondation de l'université de Kolozsvár, mais aussi la requalification de certaines académies en 1874, élevées au rang des facultés. Les académies obsolètes furent quant à elles supprimées.

Les académies de droit avaient un rôle d'appoint aux côtés des universités dans la formation juridique des hauts fonctionnaires. Leur création remontait à l'absolutisme éclairé : ce sont les six académies royales fondées par Marie-Thérèse, qui, de plus, transforma en académies les collèges et lycées juridiques confessionnels. Ce fut la première réponse institutionnelle au besoin en juristes qualifiés, tant dans le secteur privé que dans l'administration d'Etat. Les académies, où l'enseignement se faisait en deux ans en latin, préparaient alors aux métiers pratiques du droit (magistrature, barreau, administration : les fonctionnaires «moyens»), quand la Faculté se réservait la formation théorique des professeurs et celle des hauts fonctionnaires ${ }^{11}$. La situation resta inchangée jusque dans les années 1870, si ce n'est que ce réseau s'élargit à 17 académies dans les années $1860^{12}$ pour répondre aux besoins de personnel loyal et compétent, l'enseignement se faisant désormais en trois ans et en hongrois. En revanche, l'élévation de la durée des études à quatre ans, qui plaçait les académies au niveau des facultés, ne fut qu'une requalification formelle. L'accès au barreau et à la magistrature étaient réservés aux docteurs des facultés, l'université étant donc un passage obligé pour les étudiants des académies. Du fait même de leur petite taille (entre 46 et 217 étudiants par établissement en $1870^{13}$ ), elles ne pouvaient prétendre qu'à un rôle d'appoint. Il serait intéressant de constater si la proportion de diplômés des académies présents dans la haute fonction publique augmenta à partir de 1874, mais cette étude dépasserait alors le cadre du présent article. Les diplômés des académies, s’ils avaient le capital intellectuel certifié par leur diplôme, n'avaient peut-être pas le capital social de leurs homologues de la Faculté de la capitale, et leurs chances de carrière pouvaient alors être moindres.

Cette rapide présentation du réseau des écoles de droit semble nécessaire, puisque c'est là que se formait à la fois la majeure partie du personnel administratif et politique de l'époque, mais aussi et plus largement l'ensemble de l'élite sociale, que ce soit les fils de la haute noblesse désirant ainsi garantir leur capital social, ou encore les élites des nationalités, qui voyaient dans les études de droit la garantie du «contrat social d'assimilation». La formation par le droit tenait aussi une position hégémonique parmi les travailleurs intellectuels, et touchait la majorité des professions libérales et des secteurs de l'économie privée. Le droit était en effet capable de mener à presque toutes les carrières de la classe dominante, comme le montre une très belle

10. Chiffres tirés du Magyar statisztikai Szemle, 1934, cités par V. KARADY, Une «nation de juristes», op. cit.

11. A l'instar des pays germaniques, l'appellation «haut fonctionnaire» est réservée à ceux ayant une formation universitaire. Pour plus d'informations, voir W. HEINDL, Gehorsame Rebellen, Böhlau, 1991.

12. Désormais Eger, Kolozsvár, Pécs, Eperjes, Debrecen, Sárospatak, Kecskemét et Pápa ont aussi leur académie de droit : les étudiants des académies sont alors près de 1000, contre 643 étudiants dans la faculté de Budapest. voir F. ECKHART, A Jog-Es Allamtudományi Kar Története, 1667-1935, op. cit.

13. Chiffres tirés de l'Annuaire statistique hongrois, 1872, cités par V. KARADY, Une «nation de juristes», op. cit. 
étude de Victor Karády sur la formation juridique en Hongrie à la fin de l'Ancien Régime ${ }^{14}$.

\section{Contenu de la formation}

Il faut maintenant aborder le contenu de cet enseignement et les modalités de la transmission des savoirs de l'Etat. Pour des raisons de concision, cette étude se restreint au déroulement et au programme des enseignements proposés par la Faculté de droit de Budapest, dont on a vu qu'elle était le premier centre de formation de la haute administration centrale. Le caractère particulier des études de droit en Hongrie, qui apparaît dans le sur-développement du réseau des écoles de droit, peut à nouveau être appréhendé dans l'organisation des enseignements et notamment le caractère «national» de cette filière.

\subsection{Organisation de l'enseignement}

\subsubsection{Les examens d'Etat}

Depuis le début du XIXe siècle, les études de droit se faisaient en quatre ans à la Faculté, au terme desquelles l'étudiant devait passer un examen de fin d'année ou éventuellement un doctorat. Une grande réforme du néo-absolutisme fut l'introduction, en plus de la liberté d'enseignement (Lehrfreiheit), des examens d'Etat. Cette institution allemande, développée au sein des universités, lieu de savoir et de formation des intellectuels, permettait à l'Etat de recruter ses futurs cadres. Le jury était composé pour moitié de professeurs nommés par le ministère du Culte et de l'Education, l'autre moitié étant réservée à des professionnels nommés par les ministères concernés (la Justice ou l'Intérieur). Les examens d'Etat furent introduits en 1850 en Hongrie et participèrent à l'alignement de l'Université hongroise sur le modèle de Vienne.

L'organisation des examens d'Etat resta inchangée jusqu'à la première décennie suivant le Compromis de 1867 : elle consistait en deux examens, le premier, théorique, à la fin de la deuxième année, et le second pratique, de droit et de sciences de l'Etat, à la fin de la quatrième année. En revanche, la réforme de 1874 remplaça le premier examen d'Etat par des examens de fin d'année purement universitaires, et le second fut dédoublé en un examen de droit, et un de sciences de l'Etat, que les étudiants des universités comme désormais des académies (et même des académies confessionnelles) pouvaient préparer : c'est l'introduction de la bifurcation ${ }^{15}$. Ce nouvel examen d'Etat n'en gardait que le nom, puisqu'il était désormais organisé par les établissements mêmes et ne constituait plus un monopole de l'Etat. La composition du jury fut modifiée : la moitié étaient des professeurs de l'Université, le reste des professionnels nommés par ces professeurs. Alors même que l'Etat ne prenait plus part à leur organisation, les examens d'Etat restèrent pourtant le mode de recrutement de la haute administration, et le modèle du concours ne s'y substitua pas après la réforme de 1874 .

\subsubsection{Magyarisation de l'enseignement}

La réforme du ministre de l'Education Léo Thun, en 1855, poursuivit cette homogénéisation de l'enseignement supérieur dans toutes les universités de l'Empire : dorénavant, la liberté d'enseignement fut sévèrement encadrée, les programmes furent décidés depuis Vienne et marqués par la part croissante des matières «autrichiennes» (droit civil et public autrichien, statistique autrichienne, histoire du droit allemand...). Les professeurs étaient nommés par le ministre de

14. Ibidem.

15. Pour ce qui est de la bifurcation, voir 2.b. 
l'Education, qui décidait aussi de l'organisation des cours et de la tenue des examens. Il s'agit d'une véritable «germanisation» de l'enseignement. L'allemand devenait en effet la langue de l'enseignement supérieur, mais aussi la langue de l'administration, puisque la Hongrie fut incorporée dans l'Empire habsbourgeois sans reconnaissance de sa particularité. Pour la Faculté de droit, cela signifia en 1853 l'adoption de l'allemand comme langue des examens et des enseignements assurés par des professeurs dont la majorité ne parlait pas hongrois.

Un tableau de la langue de l'enseignement dans l'université de Budapest en 1857 permet pourtant de nuancer les effets de cette «germanisation» ${ }^{16}$ : sur les 25 professeurs de la Faculté de droit, 10 tenaient leur cours en hongrois, 14 en allemand, et 1 en latin (pour le droit romain). On peut ici remarquer la particularité de la Faculté de droit par rapport à celle de Lettres, où seulement 10 professeurs sur 32 tenaient leurs cours en hongrois, celle de médecine ( 2 professeurs sur 18 ) et celle de théologie (aucun professeur ne fait cours en hongrois). La magyarisation de l'enseignement s'est effectuée en effet plus tôt dans la Faculté de droit, tant dans la langue d'enseignement que dans le contenu de la formation : dès 1850, le droit administratif hongrois fut enseigné en hongrois par Emil Récsi, précurseur du rééquilibrage en faveur des matières «hongroises» qui a lieu dans les années 1860. La promulgation du Diplôme d'octobre, qui marqua aussi le retour à l'enseignement en hongrois des matières principales, fut alors l'occasion du développement des matières «nationales»: le droit privé hongrois devint une matière à examen, en 1863 recommença l'enseignement du droit public et administratif (interrompu en 1855 quand la matière fut délaissée par les étudiants car elle n'était plus suivie d'un examen) et l'histoire du droit allemand se généralisa en histoire du droit européen (il était en effet techniquement impossible de proposer une histoire du droit hongrois, l'enseignement de cette matière n'étant pas encore compilé).

Cette «nationalisation» de l'enseignement du droit rappelle la lutte pour la magyarisation durant le Vormärz ${ }^{17}$. Dans la lutte politique de la noblesse nationale contre le pouvoir impérial, la compétence juridique servit les débats qui se concentraient sur le terrain du droit public et constitutionnel. Il n'est donc pas étonnant que la magyarisation se soit radicalisée plus tôt dans l'enseignement du droit, et que cette branche ait contribué plus que tout autre filière universitaire à l'enracinement de l'idéologie nationaliste. La décennie du néo-absolutisme n'a pas pu revenir sur ces acquis, et le droit est resté la voie la plus «nationale». En ce qui concerne la formation de l'administration, on peut supposer que c'est cette loyauté orientée vers Budapest et non Vienne qui fut la cause du renvoi massif de fonctionnaires hongrois, remplacés par des fonctionnaires étrangers, allemands ou tchèques, les «hussards de Bach». Ces derniers subirent à leur tour une épuration de l'administration après le Compromis de 1867, mais de faible ampleur, qui ne remit pas en cause la particularité hongroise d'une bureaucratie ministérielle composée de $20 \%$ à $40 \%$ d'étrangers.

La «nationalisation» de l'enseignement est donc une particularité du droit en Hongrie ; elle caractérise, avec la spécialisation et la technicisation de l'enseignement, l'une des principales évolutions de la Faculté. Celle-ci s'achève en 1867 par l'affirmation du hongrois comme langue d'enseignement et d'examen. L'enseignement du droit et plus généralement la formation des fonctionnaires reflète donc les évolutions de la forme même de l'Etat, qui d'un Empire néo-

16. G. OTRUBA, «Die Universitäten in der Hochschulorganisation der Donaumonarchie. Nationale Erziehungsstätten im Vielvölkerreich 1850 bis 1914» in Student und Hochschule im 19. Jahrhundert. Studien und Materialien, Vandenhoeck \& Ruprecht, Göttingen, 1975.

17. V. KARADY, Une «nation de juristes», op. cit. 
absolutiste des années 1850, administré depuis Vienne, redevient le Royaume de Hongrie, l'une des deux entités de la Double Monarchie.

\subsection{Spécialisation et technicisation de l'enseignement}

Tout au long de cette période, l'enseignement du droit se rationalisa dans l'idée de former au métier de fonctionnaire, et non plus de délivrer un savoir général. L'université s'adapta à la nécessité de professionnaliser la formation de la haute administration.

Cette nécessité prit avant tout la forme d'une séparation progressive de l'enseignement de l'administration par rapport à la formation purement juridique. Dès 1848 émergea le projet de séparer la Faculté de droit en deux Facultés différenciées, pouvant permettre l'autonomisation de la formation de l'administration. Mais c'est finalement l'idée du lien organique de «la formation par les deux sciences» (két tudományos képzés) qui l'emporta. En 1855, la Faculté de droit prit alors son nom officiel de Faculté de Droit et de Sciences de l'Etat (Jog-és Allamtudomanyi Kar), sur le modèle de la Faculté de Vienne. L'autonomisation du champ administratif se poursuivit pourtant, et la séparation de la justice et de l'administration en 1869 eut pour conséquence, dans l'Université, l'introduction de la bifurcation (1874). Le dédoublement de l'examen d'Etat en un examen de droit et un de sciences de l'Etat, ainsi qu' un an plus tard celui du doctorat (toujours en droit et en sciences de l'Etat), vinrent compléter cette séparation de l'administration et de la justice.

Cette autonomisation se doublait d'une spécialisation de l'enseignement : on assista à une multiplication des chaires et des professeurs, avec l'introduction d'un enseignement plus technique (droit de la finance et des échanges, étude des procédures, etc...) à côté duquel persistaient des matières plus classiques comme le droit romain (complété par l'étude des Pandectes et encore souvent enseigné en latin), l'Encyclopédie (introduite en 1850), et les matières historiques, dotées d'un poids considérable ${ }^{18}$. C'est que la haute administration devait avoir une formation de «généralistes» de l'administration, contrairement à la formation essentiellement technique des échelons inférieurs, formés dans les académies. Dans ces académies d'ailleurs, le droit romain ne fut pas enseigné avant 1874 , au moment de la requalification sur le modèle des facultés.

En ce qui concerne l'enseignement des matières administratives plus précisément, on peut observer le même effort de spécialisation et de technicisation des savoirs de l'Etat. L'enseignement spécial de l'administration à l'université en Hongrie est une tradition qui remonte à la création de la chaire de politique et de sciences camérales «Politico- Cameralis» en 1769. Sur le modèle autrichien de 1752, cette chaire fut ouverte dans la Faculté de philosophie, mais bientôt rattachée à la Faculté de droit : en 1777 pour la Hongrie, et seulement en 1784 pour l'Autriche. C'est alors l'âge d'or des sciences camérales, enseignées en Hongrie à travers le filtre de Vienne par l'intermédiaire des manuels de Joseph von Sonnenfels. Le Ratio Educationis de 1777 affirmait en effet le rôle de l'Université dans le formation des hauts fonctionnaires : c'est pourquoi les sciences camérales y furent enseignées à raison de dix heures par semaine pendant deux semestres et devinrent en 1787 la première matière non-juridique sanctionnée par

18. Pour consulter les emplois du temps et le catalogue des matières enseignées, voir I. ARCZT, A „Politika tudományok“ oktatása a Budapesti Egyetem (ELTE) Jogi Karán és elő dintézményeiben : A „Politico-camerális“ -, a „Politikai tudományok“ - és a „Politikai“ (Alkotmányi és közigazgatási) Tanszék története 1769-1948, Rejtjel, Budapest, 2004, ainsi que la somme de F. ECKHART, A Jog-és Allamtudományi Kar története, 1667-1935, op. cit. 
un examen. Cette importance accordée aux sciences camérales souleva alors des oppositions selon lesquelles l'Université serait «dégradée» au rang d'institut de formation du personnel de l'Etat, une Beamtenuniversität.

Le second Ratio Educationis de 1806 signe la fin de cet âge d'or et le début de la séparation des sciences camérales dans leurs trois branches traditionnelles : la «politique» (politia, Poli$z e y)$, la finance et l'économie. Cette séparation sera définitive en 1848 au moment de la fin de l'enseignement des sciences camérales en Hongrie, perçues comme un produit de l'absolutisme germanique, lequel était justement remis en cause par la guerre d'indépendance. C'est alors la chaire de «sciences politiques», inaugurée en 1850, qui prit sa succession, après l'autonomisation de l'enseignement de l'économie et de la finance. Dans les années 1860, l'abandon de l'enseignement de Sonnenfels (enseigné jusque là) ainsi que la publication de manuels d'enseignement rédigés par les professeurs hongrois peuvent se comprendre comme une marque de l'autonomie retrouvée.

En 1873, la création de la chaire de «politique» (c'est-à-dire de politique constitutionnelle et administrative) prit le relais dans l'enseignement de l'administration. Cette dernière étape de la spécialisation de l'enseignement est à resituer dans le cadre d'un enseignement de plus en plus poussé des matières administratives au détriment des matières juridiques pures dans la Faculté de droit et de sciences de l'Etat. Cette logique répondait au nombre croissant d'inscrits dans la voie des sciences de l'Etat, qui comptaient, nous l'avons vu, un tiers des doctorants au tournant du siècle.

\subsection{Limites de l'enseignement}

La spécialisation et la rationalisation de la formation de l'administration ne doivent pourtant pas occulter les failles du système de l'enseignement supérieur de la seconde moitié du XIXe siècle. Si l'enseignement du droit se modernisait, la Faculté, elle, restait pourtant la plus conservatrice de l'université (au niveau notamment de l'introduction des séminaires, de l'entrée des femmes dans l'université, ou encore de l'admission des juifs ${ }^{19}$ ). De plus, le déroulement pratique des études, le fort taux d'absentéisme et le faible niveau des étudiants tendaient à minimiser les apports d'une formation, quand bien même elle était destinée à répondre aux exigences de qualification des hauts fonctionnaires d'un Etat moderne.

En effet, le système de la liberté d'enseignement entraîna une dégradation du niveau des étudiants dès les années 1860. C'est dans la Faculté de droit qu'on observe les moins bons résultats, le plus grand nombre de redoublements, d'échecs aux examens (30\%, que ce soit aux examens de fin d'année ou aux examens d'Etat ${ }^{20}$ ), et par voie de conséquence, la plus longue durée des études (il n'est pas rare de faire les quatre années requises en huit, neuf, voire dix ans). Le fort taux d'absentéisme ( $80 \%$ à $90 \%$ pour les inscrits dans les deux dernières années de droit ${ }^{21}$ ) a inspiré, pour ces pratiquants de l'école buissonnière, le surnom de «juristes champêtres» (mezei jogászok). L'altération des exigences universitaires ainsi que le caractère libéral du régime des études font du droit une culture mondaine plus que scientifique, attirant un public

19. Pour ce qui est des étudiants juifs, exceptionnellement admis à l'université à partir de 1782, ils purent dès 1849 se présenter au doctorat de droit civil (doctor iuris civilis), mais n'obtinrent le droit de passer l'examen de droit canon pour être „,doctor utriusque iuris“ qu'à partir de 1874, date à laquelle leur égalité dans l'université est véritablement complète.

20. A. LADANYI, A magyarországi felső oktatás a dualizmus kora második felében, op. cit.

21. Ibidem. 
étudiant séduit par la relative facilité des études et prêt à de moindres investissements intellectuels ${ }^{22}$. Cette réalité des études de droit décrédibilise les apports de la formation universitaire dans la formation de la haute bureaucratie et laisse supposer d'autres modes de formation (il n'existait pourtant pas de stages pratiques sur le modèle du référendariat prussien) ainsi que d'autres mécanismes de recrutement, moins regardants sur la qualité du diplôme.

\section{Les tentatives de rationalisation de la fonction publique et leurs conséquences sur la formation}

Comme nous l'avons vu, l'enseignement des sciences de l'Etat suivit une logique de spécialisation d'un savoir de plus en plus technique. Cette logique participait de la formation d'un véritable métier de l'administration, qui passait par la transmission d'un savoir (savoir-faire) prérequis, ainsi que par la codification du statut de fonctionnaire. Il ne s'agissait plus d'une vocation d'aristocrates appelés par le souverain, mais d'une fonction publique rationnelle administrant un Etat moderne. La rationalisation passait avant tout par la codification de la qualification, c'est-à-dire par la mise en place d'une logique selon laquelle un poste ne pouvait être occupé que par quelqu'un pouvant certifier sa compétence par le diplôme adéquat.

Cette exigence d'une qualification ne se codifia que progressivement. Sous Marie-Thérèse, on ne demandait des hauts fonctionnaires qu'un passage à l'université pour acquérir «la connaissance des lois». Dans sa «lettre du pasteur» (Hirtenbrief, pásztorlevél), Joseph II exigeait surtout des qualités morales, ainsi qu'un «esprit clair». L'ordonnance de 1787 était plus explicite en ce qu'elle requérait des études de droit «menées à terme» pour les hauts postes de l'administration centrale. Il s'agissait pour l'époque de la sélection la plus aboutie sur le critère du droit. Un siècle plus tard, en 1870, une loi sur les «spécialistes de la politique» (comprendre : de l'administration au sens large) énumérait des critères généraux : avoir au moins 22 ans, un casier judiciaire vide et aucune poursuite légale en cours. Pour les fonctionnaires de l'administration centrale, ainsi que certains de l'administration locale, un diplôme de droit était exigé, sans codification systématique ni autre prérequis pour l'administration locale. Peu de postes exigeaient donc nommément une qualification, et celle-ci restait très générale («examen de droit»).

La loi 1. de 1883, dite loi de «qualification» (minôsítés), répondait à ce besoin de codification systématique de la plupart des postes de l'administration centrale et locale. Pour les hauts fonctionnaires de l'administration ministérielle, on exigeait désormais un examen d'Etat, de droit comme de sciences de l'Etat, ou bien un doctorat, encore une fois de droit ou de sciences de l'Etat, ou encore un diplôme d'avocat. A cette formation théorique, il était prévu d'ajouter une formation pratique, mais la mise au point d'un examen d'Etat pratique ne se fit qu'en 1937. On remarque toute l'ambiguïté de cette exigence de formation, qui reconnaissait aussi bien la formation proprement administrative par les sciences de l'Etat que celle purement juridique couverte par le diplôme d'avocat.

La loi prévoyait aussi des cas de double qualification pour les ministères spéciaux, tels la Justice qui, pour certains postes techniques, exigeait un diplôme de juge ou de magistrat en plus de l'un des deux examens d'Etat; ou le département de la Santé du ministère de l'Intérieur qui pour certaines fonctions embauchait aussi des diplômés de le faculté de Médecine; ou encore au Cadastre, dépendant du ministère des Finances, qui embauchait des ingénieurs. Une clause prévoyait en plus des dérogations exceptionnelles, selon lesquelles, dans les ministères

22. V. KARADY, Une «Nation de Juristes», op. cit. 
techniques, il était possible d'embaucher le titulaire d'un diplôme dans le domaine concerné (et non en droit), à condition qu'il passe un examen de droit. Cette loi de qualification marquait donc le «privilège des juristes», et non le «monopole des juristes» (Juristenmonopol) comme la loi de 1879 en Prusse.

Dans son application, cette loi ne mit pas fin du jour au lendemain aux pratiques de recommandation (Protektion) et ne fut pas non plus systématiquement appliquée. Là encore, des statistiques manquent pour évaluer les conséquences sur le recrutement des hauts fonctionnaires. Cette loi marquait pourtant une considérable avancée dans la tentative de rationalisation de l'administration. Elle participait à l'élaboration d'une Dienstpragmatik, d'une «pragmatique du service public», c'est-à-dire du «métier» de fonctionnaire, puisqu'elle visait à la codification du statut du fonctionnaire, de ses droits et de ses devoirs, de sa carrière (de la nomination au renvoi, en passant par les promotions et les mutations), de son existence matérielle (salaire et retraite), et plus largement, d'une éthique du service public. D'autres lois allèrent dans le sens de l'élaboration de cette pragmatique, mais celle-ci ne vit pas le jour en Hongrie avant la première guerre mondiale.

\section{Les revendications des non-juristes à la fin du XIXe}

Il faut maintenant aborder un dernier point sans lequel ce tableau ne serait pas complet, à savoir les revendications des non-juristes qui réclamaient un rôle plus important dans l'administration. Ces revendications furent accompagnées, à la fin du XIXe siècle, par la critique de la formation unilatéralement juridique de l'administration, le droit apparaissant finalement comme une formation trop classique, voire archaïque. Au contraire, ces revendications en appelaient à une administration de plus en plus spécialisée, administrée par les spécialistes mêmes, et non plus par ces «généralistes de l'administration» que sont les juristes. Ce discours ne doit pourtant pas occulter la présence tout au long de la période (mais difficilement quantifiable) de non-juristes au sein de l'administration, soit qu'ils aient eu une autre formation (et dans ce cas il serait intéressant de connaître laquelle, particulièrement avant la loi de 1883 qui d'une certaine manière «autorise» les non-juristes à entrer dans l'administration), soient qu' ils n'aient pas de diplôme (ce qui pourrait alors permettre d'estimer l'ampleur des pratiques de népotisme et de protection).

Attardons-nous tout d'abord sur les revendications des spécialistes soucieux d'obtenir un rôle dans l'administration de leur spécialité. Il s'agit des médecins, qui par exemple se plaignaient en 1910 de ce qu'un juriste ait repris la direction du département de la Santé au ministère de l'Intérieur ${ }^{23}$, mais aussi des vétérinaires, dont le service fut étatisé en 1900. Les agronomes enfin se plaignaient de ce qu'en 1914, sur douze directeurs de cabinet au ministère de l'Agriculture, seuls deux aient fait des études d'agronomie. Ce chiffre montre à la fois la présence de non-juristes aux plus hauts postes de l'administration, mais aussi la faible proportion de ces derniers dans un ministère aussi «technique» que celui de l'Agriculture.

Les ingénieurs forment un cas particulier puisqu'ils revendiquaient la compétence générale d'administrer qui restait jusqu'alors l'apanage des juristes. Rappelons qu'en France, au contraire, c'était les élèves-ingénieurs des Corps des Grandes Ecoles d'ingénieurs qui détiennent les hautes fonctions et qu'en Allemagne aussi, le modèle des Beamteningenieure, des ingénieurs-fonctionnaires, gagnait du terrain. En Hongrie, dès 1867, les ingénieurs reçurent

23. A. HENCZ, Felsőfokú közigazgatási szakemberképzés Magyarországon, 1848- 1948, Budapest, 1981. 
l'autorisation de travailler dans l'administration locale, après avoir passé un examen de droit. Ils y exerçaient pourtant des fonctions de moindre importance que les juristes. De même, la loi de qualification de 1883 ne leur proposait que des postes «techniques» (müszaki). Les ingénieurs réclamèrent alors l'égalité avec les juristes pour tous les postes, ainsi que leur prééminence pour les postes techniques. Leur argument se fondait sur la séparation de la justice et de l'administration en 1869, et donc sur le fait qu'il n'était plus nécessaire que le droit soit l'unique formation de l'administration. Mais cette demande de parité des ingénieurs avec les juristes paraît encore irréaliste au tournant du siècle, au regard des écarts entre la production de juristes et d'ingénieurs : chaque année, 7000 juristes étaient formés en Hongrie contre 1400 techniciens (pour l'Autriche, l'écart est bien moindre : 10500 juristes contre 9000 techniciens). On remarque au passage une nouvelle fois la surproduction de juristes en Hongrie.

Il est à noter qu'à partir de 1914, des cours d'économie furent tenus à l'Université Polytechnique de Budapest qui, au terme d'un an d'un tel enseignement, proposait de former des «ingénieurs culturels» (kulturmérnök). Mais la réglementation d'un éventuel doctorat d'économie, préparant les ingénieurs à l'administration, fut interrompue par le déclenchement de la première guerre mondiale.

\section{Conclusion}

Le «privilège des juristes» dans l'administration fut de règle en Hongrie, depuis que MarieThérèse assigna à l'université l'objectif de former l'appareil d'Etat. Si initialement cet objectif fut dévolu à la faculté de Philosophie où étaient alors enseignées les sciences camérales, il devint rapidement l'apanage de la faculté de droit (rebaptisée en 1855 Faculté de Droit et de Sciences de l'Etat). La loi de qualification de 1883 vint consacrer ce «privilège des juristes». Le métier de l'Etat s'appuie sur un savoir juridique.

Entre-temps, des réformes furent mises en oeuvre dans la Faculté de Droit afin de moderniser et spécialiser la formation des futurs cadres de la bureaucratie. Celle-ci était orientée soit depuis Vienne (rappelons la soumission quasi-totale de l'université durant la décennie du néoabsolutisme), soit depuis Budapest (à partir des années 1860, mais surtout après le Compromis de 1867), toujours dans le sens d'une plus grande rationalisation. C'est cette formation particulière par le droit qui a contribué à l'émergence d'une bureaucratie d'Etat hongroise, dans l'esprit d'une culture juridique porteuse de l'idéologie nationale et libérale. Cette culture commune des élites, ce consensus autour du langage commun du droit a fait de la Hongrie une «nation de juristes».

Les désastres de cette surproduction de juristes se firent sentir après la première guerre mondiale, la défaite et la tourmente de l'après guerre, rendues tragiques par les pertes territoriales que connut la Hongrie. La contraction du territoire alla de pair avec l'expulsion des fonctionnaires dans les pays successeurs, leur loyauté étant devenue suspecte. Cela signifia pour la Hongrie un afflux de fonctionnaires : entre 1918 et 1924, le pays accueillit 16000 membres du secteur public et 19000 employés des chemins de fer, dans un marché déjà saturé ${ }^{24}$. Le pouvoir d'achat des fonctionnaires fut diminué de 4/5e (en comparaison, celui des ouvriers fut diminué «seulement» de moitié). Les fonctionnaires connurent donc un déclassement et un sentiment d'insécurité dont les premières victimes furent les minorités et les marginaux, en particuliers les

24. Chiffres tirés de Budapest székesfövaros statisztikai közleményei, cités par V. KARADY, Une «nation de juristes», op. cit. 
Juifs sur-représentés dans les métiers intellectuels accessibles après une formation supérieure. La première mesure antisémite toucha donc la formation universitaire : la loi du numerus clausus de 1920 limita l'accès à l'université selon des quotas relatifs à l'appartenance ethnique, espérant ainsi freiner la surproduction de juristes. S'il faut certes en minimiser l'application, notamment dans la Faculté de Droit ${ }^{25}$, cette mesure marqua tout de même la mort indigne de la «nation de juristes».

25. De 1920 à 1925, c'est-à-dire durant les premières années d'application de la loi, la proportion des étudiants juifs dans la Faculté de droit dépasse toujours le double du quota prescrit (6\%). De plus, ils avaient toujours la possibilité de se rabattre sur les facultés de province et les académies de droit, qui avaient du mal à remplir leur contingent d'étudiant face à la concurrence de la capitale, et obtenaient ainsi des dérogations. La Faculté de médecine de l'Université de Pécs connait une situation plus particulière encore, puisque de 1920 à 1925, la moitié de ses étudiants étaient juifs. V. KARADY, I. KEMENY, Antisémitisme universitaire et concurrence des classes. La loi du Numerus Clausus, Actes de la recherche en sciences sociales, 34, 1980. 